\title{
Neurología Argentina
}

www.elsevier.es/neurolarg

Historia y humanidades

\section{Saber y no poder. Tratamiento agudo del accidente cerebrovascular y políticas sanitarias. Una mirada situada desde el Comité de Bioética, Hospital de Área Programa El Bolsón, Río Negro}

\author{
Susana Candia ${ }^{a}$, Claudia Melihual $^{b}$, Marcelo Muscillo $^{a}$, Carla Roggero $^{b}$, Pablo Ruiz $^{a}$, \\ Karina Swain ${ }^{a}$, M. Alma Tozzini ${ }^{a}$ y Sebastián Villate ${ }^{b, *}$ \\ a Miembros externos pertenecientes al Comité de Bioética del Hospital de Área Programa El Bolsón, El Bolsón, Río Negro, Argentina \\ b Miembros internos pertenecientes al Comité de Bioética del Hospital de Área Programa El Bolsón, El Bolsón, Río Negro, Argentina
}

INFORMACIÓN DEL ARTÍCULO

Historia del artículo:

Recibido el 4 de noviembre de 2018

Aceptado el 23 de enero de 2019

Palabras clave:

ACV

Bioética

Derechos humanos

Keywords:

Stroke

Bioethics

Human rights

\section{R E S U M E N}

Introducción: Se presenta un dictamen del Comité de Bioética perteneciente al Hospital de Área El Bolsón sobre posibles tratamientos del accidente cerebrovascular (ACV).

Objetivo: Analizar desde el paradigma bioético centrado en Derechos Humanos y en el contexto de la Provincia de Río Negro la falta de implementación del tratamiento agudo del ACV.

Desarrollo: La ausencia de marco normativo posiciona a los aspectos bioéticos en un lugar de máxima importancia para con los usuarios de salud.

Conclusión: La confianza en la relación médico-paciente podría dañarse debido a la controversia entre el marco normativo y un conocimiento que otorgaría una mejoría al paciente.

๔ 2019 Sociedad Neurológica Argentina. Publicado por Elsevier España, S.L.U. Todos los derechos reservados.

To know and to cannot. Acute treatment of stroke and health policies. A Bioethics Committee perspective, Program Area, El Bolsón Hospital, Río Negro province

A B S T R A C T

Introduction: We present a report of the Bioethics Committee of the Hospital of El Bolsón about possible stroke treatments.

Aim: To analyze the absence of the acute stroke treatment implementation from a bioethical perspective based on Human Rights in the context of the Province of Río Negro, Argentina. Discussion: The absence of a law in this respect turns bioethical aspects into a matter of utmost relevance for healthcare service users.

\footnotetext{
* Autor para correspondencia.

Correo electrónico: sebastianvillate@hotmail.com (S. Villate).

https://doi.org/10.1016/j.neuarg.2019.01.004

1853-0028/@ 2019 Sociedad Neurológica Argentina. Publicado por Elsevier España, S.L.U. Todos los derechos reservados.
} 
Conclusion: The trust in the doctor-patient relationship could be damaged due to conflicts between legal rules and medical knowledge that would mean an improvement for patients.

@ 2019 Sociedad Neurológica Argentina. Published by Elsevier España, S.L.U. All rights

reserved.

\section{Introducción}

El escrito que presentamos a continuación es el texto completo del Dictamen elaborado desde el Comité de Bioética de El Bolsón a los fines de reflexionar sobre las posibilidades del tratamiento de los accidentes cerebrovasculares (ACV) en la localidad y la provincia.

La propuesta llegó de la mano de uno de nuestros integrantes, especialista en la materia, y rápidamente el dilema ético que el caso presentaba nos encontró a todos reflexionando sobre el particular.

Entendimos que la apuesta resultaría algo disruptiva, lo que nos alentó, en principio, a compartirla con otros comités de bioética que dedicaron su valioso tiempo para realizarnos una devolución, así como de otros especialistas en bioética con quienes pudimos compartir nuestras premisas filosóficas de construcción de la problemática y el objeto. Si bien el planteo ya clásico en bioética respecto de la eventual dicotomía beneficencia/medios escasos sobrevoló inicialmente la parte exploratoria de nuestras reflexiones, decidimos centrar nuestro análisis en el paradigma referente a los Derechos Humanos, hoy día superador del primero.

\section{Comentarios}

\section{Planteo de la problemática}

Los accidentes cerebrovasculares (ACV) representan una de las principales causas de mortalidad y discapacidad en nuestro país $^{1,2}$.

Desde el año 1996 existe medicación aprobada por ANMAT para el tratamiento agudo del ACV; pese a esto, menos del $10 \%$ de la población mundial lo recibe, y en Argentina solo el 1\% consigue dicho tratamiento ${ }^{2-4}$.

No realizar el tratamiento agudo del ACV a un usuario no solo implica privarlo de una terapéutica valiosa, sino que también genera costos al sistema de salud. En el artículo «Análisis de gastos públicos en la provincia de Río Negro debido a accidentes cerebrovasculares. Una razón más para iniciar el tratamiento fibrinolítico» ${ }^{5}$ se indican algunos de los gastos que afronta el Ministerio de Salud provincial al abordar el tratamiento de pacientes que sufrieron un ACV. Se especifican los insumos destinados para estudio, internación y rehabilitación, mostrando estimaciones donde el costo del fibrinolítico se asemeja a los gastos generados por internaciones prolongadas y la rehabilitación solo del primer año luego de un $\mathrm{ACV}^{5}$. El estudio no incluyó entre sus montos las pensiones, así como tampoco los años perdidos de trabajo ni los gastos de medicación, lo cual aumentaría aún más el total. Esto demuestra que, en función de los costos, la utilización del fibrinolítico implicaría un costo incluso menor de aquel que igualmente el Estado eroga para el tratamiento, internación y rehabilitación de los pacientes con esta patología, mientras que la calidad de vida del paciente se ve severamente deteriorada.

La Provincia de Río Negro presenta 708.799 habitantes asistidos sanitariamente por medio del sistema de salud público y privado. El primero se organiza según niveles de complejidad; las ciudades de Bariloche, General Roca, Viedma y Cipolletti presentan los hospitales de máxima complejidad, llamados Hospitales Zonales o de complejidad tipo VII. Estos cuentan con unidades de cuidados intensivos, tomógrafos en la institución, neonatología y diversas especialidades médicas. Por debajo de estos se encuentran hospitales con niveles VI, III, y diversos puestos sanitarios.

El Hospital de Área Programa El Bolsón es un hospital complejidad VIb, y por lo tanto tiene las especialidades básicas (ginecología, clínica médica, cirugía y pediatría), a las cuales se agregan infectología, cardiología, traumatología, endocrinología y neurología. Concurren ocasionalmente especialistas de diagnóstico por imágenes, urología, nefrología, neonatología y oncología. Dispone también de salud mental, servicio social, rehabilitación kinésica y fonoaudiológica.

Dicho nosocomio cuenta con 41 camas en total, distribuidas en una sala general y una de materno-infancia, esta última con una sala de preparto y de reanimación neonatal; dispone de una unidad de cuidados intermedios (UCI) con 4 camas y 2 enfermeros. Posee guardia pasiva de rayos y laboratorio las $24 \mathrm{~h}$ y activas los fines de semana. Presenta 2 quirófanos, una sala de partos y hemoterapia. Las tomografías de urgencias se realizan a través de contrataciones a empresas privadas locales; las programadas se ejecutan en el Hospital Zonal de El Bolsón.

El sistema de guardia se divide en una general y una de clínica médica, ambas activas. La primera es asistida por médicos generalistas en su mayoría y la segunda se desarrolla en el espacio físico de la UCI. Dicho hospital tiene como centro de referencia el Hospital Zonal de Bariloche, a $160 \mathrm{~km}$ de distancia.

Si bien los hospitales zonales de Río Negro se encuentran gestionando su posible implementación, aún no se dispone de unidades para el tratamiento agudo del ACV funcionando en los hospitales públicos, pese a contar con la infraestructura y con los recursos humanos necesarios para la realización de dicho procedimiento ${ }^{6}$.

Esto ejemplifica el dilema que nos ocupa en esta recomendación, al existir una evidencia científica que queda relegada por las políticas de salud pública. El tratamiento es efectivo ${ }^{7-9} \mathrm{y}$ puede realizarse dependiendo de la complejidad hospitalaria; sin embargo, no se lleva a cabo en la actualidad en hospitales públicos rionegrinos y tampoco forma parte del Programa Médico Obligatorio de la Nación. 


\section{Definiciones teóricas}

- Accidente cerebrovascular (ACV): síndrome clínico de desarrollo rápido debido a una perturbación focal de la función cerebral de origen vascular y de más de 24 h de duración ${ }^{10}$.

- Accidente cerebrovascular isquémico: tipo de ACV producido por la obstrucción de una arteria cerebral.

- Tratamiento agudo del ACV/Tratamiento fibrinolítico o trombolítico del ACV: consiste en la desobstrucción de una arteria cerebral mediante drogas fibrinolíticas o desobstrucción mecánica que se realiza entre las 3 a $6 \mathrm{~h}$ de iniciados los síntomas.

- Trombólisis o fibrinólisis: consiste en la disolución de un coágulo que obstruye el lecho sanguíneo.

- Procedimiento del tratamiento endovenoso agudo del ACV: consiste en la desobstrucción de una arteria cerebral mediante drogas fibrinolíticas antes de las $4 \mathrm{~h} 30 \mathrm{~min}$ de iniciados los síntomas, y la desobstrucción mecánica dentro de las $6 \mathrm{~h}$. Se administran 0,9 mg/kg de peso del paciente, el 10\% de dicha cantidad mediante bolo y el restante en la hora siguiente; la máxima dosis aplicada es $90 \mathrm{mg}$.

- Factor activador del plasminógeno tisular (alteplase, rtPA): fármaco utilizado para el procedimiento. Su función consiste en activar el paso de plasminógeno a plasmina hidrolizando así las redes de fibrina, con lo cual canaliza nuevamente el vaso $^{11-13}$.

\section{Marco normativo}

- No existen hasta el momento normativas nacionales o provinciales acerca de la administración del tratamiento agudo (fibrinolítico/trombolítico) de los accidentes cerebrovasculares isquémicos en nuestro país. Tampoco el alteplase (fibrinolítico), así como el tratamiento anteriormente citado, forman parte del Programa Médico Obligatorio (PMO) de emergencia.

- En la Honorable Cámara de Diputados fueron presentados, por los diversos bloques, diez proyectos de ley entre los años 2013 y 2017; solo dos de estos no han caducado aún. Sin embargo, ambos tienen como objetivo la declaración de interés nacional sobre la prevención de los ACV y no el tratamiento. La Dirección Nacional de Enfermedades Crónicas no Transmisibles del Ministerio de Salud de la Nación no tiene programas en vigencia ni se tiene conocimiento que se encuentre entre sus objetivos próximos la incorporación del tratamiento agudo del ACV.

\section{Discusión}

El punto de partida al analizar una problemática bioética consiste en definir el aspecto ético de la demanda; es decir, ¿existe un problema ético? y/o, en forma contrapuesta, ¿existe una normativa clara que defina las acciones a seguir?

Puntualmente el caso presentado no reviste de normativas nacionales ni provinciales acerca de dicho tratamiento. Existe la ley 26529 acerca de los Derechos del Paciente en su Relación con los Profesionales e Instituciones de la Salud; uno de sus artículos establece el derecho a recibir la información sanitaria necesaria, vinculada a la salud del usuario; por lo tanto, podemos entrever aquí una dificultad de índole ético.

Según el párrafo anterior, queda establecido que el paciente tiene derecho al acceso a la información; entonces, al interrogarnos acerca de la existencia de un tratamiento para el ACV, o si es posible hacer algo para revertir las secuelas; la respuesta sería: «sí, pero no es posible». Sin duda, al posicionarnos como pacientes que presentan lesiones posiblemente definitivas, la siguiente pregunta consistiría en: ¿y por qué no se puede hacer?

Frente a la falta de programas nacionales para su incorporación en los distintos ámbitos de salud (hospitales públicos/prepagas), como así también ante la ausencia de legislación al respecto, asumimos que esta pregunta no ha despertado interés aún por parte de las autoridades del Ministerio de Salud.

\section{Aspectos éticos}

\section{Presentación}

En términos de la relación entre personas, y entre estas y cualquier exterioridad física, siguiendo la línea tendida por autores como Agamben ${ }^{14}$, Lévinas ${ }^{15}$ o Derrida ${ }^{16}$, hemos intentado en dictámenes anteriores reforzar la hipótesis de que el mejor acceso para entrever los problemas de índole ético, tanto en referencia a una ética general como a una aplicada (bioética), tendría lugar antes que el conocimiento, antes que la palabra establezca los límites de esas relaciones ${ }^{a}$. Es decir, que a partir de un entretejido de relaciones previas a la palabra se configura el ámbito de la ética, que luego habilita el texto, la ley que, en definitiva, sostiene la forma epistémica. Pero el conocimiento que de este modo la palabra inaugura ¿resulta totalmente independiente de ese plano ético?

El caso que hoy nos ocupa se presenta particularmente crítico, puesto que se trataría de establecer hasta dónde es posible des-conocer lo conocido, de qué modo hacerlo, y determinar si una posibilidad tal alteraría la relación ética que ha hecho posible ese conocimiento, que le ha dado sentido. Es decir, interrogarnos acerca de si ese conocimiento, una vez construido, resulta absolutamente externo a aquella instancia previa que propusimos como ámbito ético, o vuelve hacia allí de un modo u otro, involucrando nuevamente a las personas y las cosas que habitan ese ámbito, y de qué forma. En los términos concretos que nos proponemos indagar, cuestionarnos acerca de si podría un/a profesional de la salud ocultar o disimular un conocimiento sin generar una tensión de índole profundamente ético.

\footnotetext{
a Por ejemplo, el ejercicio, claramente psicoanalítico, que ensaya Derrida (1989), a partir de aquel otro que en los trabajos de Heidegger (y por dificultades en la traducción entre idiomas) se ha dado en llamar de-construcción, puede considerarse como una muestra de ese acceso $^{16}$.
} 


\section{Desarrollo}

Conocimiento y violencia

Como lo han señalado tantos pensadores, pero muy especialmente Foucault ${ }^{17}$, Bourdieu ${ }^{18}$ y antes que ellos Nietzsche, el conocimiento se presenta claramente, y en el más profundo sentido filosófico, como una relación de poder que apenas disimula la violencia que conlleva. La prioridad reclamada entonces en favor de la ética trataría de subsanar, en lo posible, esa violencia inevitable ${ }^{b}$.

La palabra del otro/a, ante la duda cartesiana, plasmada luego en la ley, nos asegura que estamos en el mundo, en ese mundo del que hablamos. Nos asegura que somos reales, igual que toda esa exterioridad que sentimos alrededor nuestro. La fijación que la palabra implica, es decir, la correspondencia entre la palabra y aquello que queremos designar con ella, nace de una complicidad previa entre las personas, sostenida desde una instancia primera y emocional ${ }^{\mathrm{c}}$, y es la que otorga a esas personas, involucradas en ese comercio, su identidad, su certeza de ser. El conocimiento, con sus definiciones y sus leyes, es parte de esa negociación.

\section{Conocimiento y ética}

Sin embargo, la univocidad que la ciencia de Occidente impone como conocimiento universal no es la única perspectiva posible. Otras miradas han preferido ubicar la verdad en una instancia más profunda que la que implica esa sola correspondencia entre significante y significado que hemos descripto. «Lo verdadero es lo no-dicho, que solo en un decir riguroso y adecuado, continúa siendo lo no-dicho que es.» Desde posiciones como estas, para las cuales la cuestión semántica no agota la discusión filosófica sobre la verdad ${ }^{\mathrm{d}}$, la ética vuelve a ser relevante pero no solamente en referencia a juicios de valor, sino también con respecto a aquellos de certeza.

De este modo, el conocimiento, que es posible a partir de la condición ética, refuerza y recrea esta condición, y el ámbito ético, aun considerado como prioritario, no resulta independiente del ámbito textual, por lo que des-conocer lo conocido no resultaría inocuo.

La verdad, considerada en referencia a una semántica, se opone a la falsedad (decir de algo aquello que no es); en cam-

\footnotetext{
b Según Foucault, para Nietzsche «el conocimiento solo puede ser una violación de las cosas a conocer y no percepción, reconocimiento, identificación a o con ellas $»^{17}$.

c «No es la mediación del signo la que hace la significación, sino que es la significación (cuyo acontecimiento original es el cara a cara) la que hace posible la función del signo» ${ }^{19}$.

d Lacan objeta la «definición de una sociedad como colección de individuos»: Esto «es hacer entrar por una puerta muy diferente la incidencia de la verdad como causa e imponer una revisión del proceso de causalidad». «Si toda causalidad viene a dar testimonio de una implicación del sujeto, no hay duda de que todo conflicto de orden sea puesto en su cuenta.» Aquí se ve cómo el sujeto, como tal, es el que ordena la exterioridad que siente, el que «acomoda la existencia». El sujeto es responsable ante los demás de ese orden, debe dar cuenta de él. No hay nada de casual en las palabras que emplea Lacan. Cuando dice que el conflicto de orden corre por cuenta del sujeto señala una implicación, una culpabilidad, una deuda material, del mismo modo que lo ha propuesto Nietzsche, llega al asunto del deber moral a través de una cuenta material.
}

bio, llevada hacia el plano prioritario de la ética se opone a la mentira, al engaño, que más allá de la certeza de la correspondencia semántica afecta directamente a la relación entre personas, pues supone presentar como verdadero aquello que se sabe falso. La persona que cree conocer la verdad, más allá de la relatividad de ese conocimiento, no puede esconderla o negarla sin que eso altere su relación con las otras personas a partir de las cuales, y gracias a las relaciones establecidas entre ellas, esa verdad ha sido posible. En función de ello, y en referencia al tema que estamos analizando, ya podríamos adelantar que no podría una normativa obligarnos a ocultar un conocimiento, obligarnos a engañar. Quien se encuentra en posesión de una certeza, ya provenga esta de una revelación, de un hallazgo, de una enseñanza o de una experiencia propia, ya se considere o no un conocimiento positivo en el sentido occidental de tal expresión, no puede sin más ocultarla sin afectar el ámbito ético al que esa persona pertenece y a partir del cual es lo que es. Más allá del modo en que esa certeza esté justificada, de la cuestión epistemológica, si la sociedad, en la cual esa persona encuentra su identidad mediante una dimensión ética particular, valora a esa certeza como un bien (situación que no necesariamente debe ser así), no puede luego obligarse a ninguno de los involucrados en esa relación a ocultarla o negarla sin que ello implique una situación éticamente contradictoria y violenta.

En el caso que tratamos aquí, nos gustaría arriesgar que obstaculizar la aplicación de un conocimiento que la sociedad valoraría como bueno, negando el acceso a la tecnología que ese conocimiento habilita, no podría no resultar profundamente perturbador en lo que a la ética se refiere, tanto para el/la paciente como para el equipo profesional que interviene.

\section{Conclusiones}

1. Según hemos tratado de exponer, entendemos que los procedimientos que se sugieren no están aún en las agendas sanitarias de las autoridades, siendo nuestra sugerencia en general que esta situación pueda ser contemplada, en virtud de los considerandos éticos desarrollados.

2. En un principio entendemos que la confianza en la relación médico-paciente se vería lesionada en tanto el primero estaría obligado por normativa a no ofrecer o no presentar un conocimiento que a priori habilitaría una mejoría en el paciente.

3. Por todo esto, quisiéramos sugerir que sería acertado instalar en nuestras dependencias sanitarias la consideración de estos interrogantes con miras a la implementación de estas prácticas.

\section{Conflicto de intereses}

Los autores declaran no tener conflicto de intereses.

\section{Agradecimientos}

Gratificamos especialmente a los integrantes del Comité de Bioética de la Zona Sanitaria Norte de la Provincia de Chubut y a la Mag. Gabriela Demarchi de la Universidad Nacional San 
Juan Bosco, Sede Esquel y Hospital Zonal de Esquel, Chubut, por los comentarios que ayudaron a enriquecer este dictamen.

\section{B I B LIOGR A F Í A}

1. WHO Global Infobase. World Health Organization. Disponible en: https://apps.who.int/infobase/Mortality.aspx.

2. Sposato L, Esnaola M, Zamora R, Zurru M, Fustinoni O, Saposnik G. Quality of ischemic stroke care in emerging countries: The Argentinian National Stroke Registry (ReNACer). Stroke. 2008;39:3036-41.

3. Moretti A, Ferrari F, Villa R. Pharmacological therapy of acute ischaemic stroke: Achievements and problems. Pharmacol Ther. 2015;153:79-89.

4. Balami J, Chen R, Sutherland B, Bucha A. Thrombolytic agents for acute ischaemic stroke treatment: The past, present and future. CNS Neurol Disord Drug Targets. 2013;12:145-54.

5. Villate S, Fernández M. Análisis de gastos públicos en la provincia de Río Negro debido a accidentes cerebrovasculares. Una razón más para iniciar el tratamiento fibrinolítico. Neurol Arg. 2016;8:307-9.

6. Zuin D, Nofal P, Tarulla A, Reynoso F, Ollari F, Alves Pinheiro A, et al. Relevamiento epidemiológico nacional de recursos neurológicos: presencia de centros de tratamiento del accidente cerebro vascular con trombolíticos en Argentina. Neurol Arg. 2015;7:261-5.

7. Sandercock P, Wardlaw JM, Lindley RI, Dennis M, Cohen G, Murray G. Thebenefits and harms of intravenous thrombolysis with recombinant issue plasminogen activatorwithin $6 \mathrm{~h}$ of acute ischaemicstroke (the Third International Stroke Trial [IST-3]): A randomised controlled trial. Lancet. 2012;379:2352-63.

8. Wahlgren N, Ahmed N, Dávalos A, Ford GA, Grond M, Hacke W. Thrombolysis with alteplase foracute ischaemic stroke in the Safe Implementation of Thrombolysis in Stroke-Monitoring Study (SITS-MOST): An observational study. Lancet. 2007;369:275-82.
9. Powers W, Rabinstein A, Ackerson T, Adeoye O, Bambakidis N, Becker K, et al. 2018 Guidelines for the Early Management of Patients with Acute Ischemic Stroke. A Guideline for Health care Professionals from the American Heart Association/American Stroke Association. Stroke. 2018, http://dx.doi.org/10.1161/STR. 0000000000000158.

10. Accidente cerebrovascular. Organización Mundial de la Salud. Disponible en: https://www.who.int/topics/cerebrovascular_accident/es/.

11. Sacco R, Kasner S, Broderick J, Caplan L, Connors J, Culebras A, et al. Professionals from the American Heart Association/American Stroke Association. An Updated Definition of Stroke for the 21st Century: A Statement for Healthcare. Stroke. 2013;44: 2064-208.

12. Powers W, Rabinstein A, Ackerson T, Adeoye $\mathrm{O}$, Bambakidis $\mathrm{N}$, Becker K, et al. 2018 Guidelines for the early management of patients with acute ischemic stroke. A guideline for healthcare professionals from the American Heart Association/American Stroke Association. Stroke. 2008, http://dx.doi.org/10.1161/STR. 0000000000000158.

13. Jauch E, Saver J, Adams H, Bruno A, Connors JJ, Demaerschalk $\mathrm{BM}$, et al. Guidelines for the early management of patients with acute ischemic stroke. A guideline for healthcare professionals from the American Heart Association/American Stroke Association. Stroke. 2013;44:870-947.

14. Agamben G. Profanaciones. 1. ${ }^{a}$ edición Buenos Aires: Adriana Hidalgo; 2005

15. Lévinas E. Ética como filosofía primera. A Parte Rei. Revista de filosofía. 2006;46, https://dialnet.unirioja.es/ejemplar/323516.

16. Derrida J. La escritura y la diferencia. Barcelona: Anthropos; 1989

17. Foucault M. La verdad y las formas jurídicas. Madrid: Gedisa; 1980

18. Bourdieu P. Campo de poder, campo intelectual. 5. a edición Buenos Aires: Montressor Jungla Simbólica; 2002.

19. Lévinas E. Totalidad e Infinito. $2 .^{\text {a }}$ edición Salamanca: Sígueme; 2012. p. 11-230. 